\title{
A Brief Course in Spontaneous Symmetry Breaking II. Modern Times: The BEH Mechanism
}

\section{François Englert ${ }^{* \dagger}$}

Service de Physique Théorique, Université Libre de Bruxelles, Campus Plaine, C.P.225,

Boulevard du Triomphe, B-1050 Bruxelles, Belgium E-mail: EenqLertduLb.ac.be

The theory of symmetry breaking in presence of gauge fields is presented, following the historical track. Particular emphasis is placed upon the underlying concepts.

Proceedings of the Corfu Summer Institute 2012 "School and Workshops on Elementary Particle Physics and Gravity"

September 8-27, 2012

Corfu, Greece

\footnotetext{
* Speaker.

${ }^{\dagger}$ Invited talks presented at the 2001 Corfu Summer Institute on Elementary Particle Physics.
} 


\section{Introduction}

It was known in the first half of the twentieth century that, at the atomic level and at larger distance scales, all phenomena appear to be governed by the laws of classical general relativity and of quantum electrodynamics.

Gravitational and electromagnetic forces are long range and hence can be perceived directly without the mediation of highly sophisticated technical devices. The development of large scale physics, initiated by the Galilean inertial principle, is surely tributary to this circumstance. It then took about three centuries to achieve a successful description of long range effects.

The discovery of subatomic structures and of the concomitant weak and strong interaction short range forces raised the question of how to cope with short range forces in quantum field theory. The Fermi theory of weak interactions, formulated in terms of a four Fermi point-like current-current interaction, was predictive in lowest order perturbation theory and successfully confronted many experimental data. However, it was clearly inconsistent in higher order because of uncontrollable quantum divergences at high energies. In order words, in contradistinction with quantum electrodynamics, the Fermi theory is not renormalizable. This difficulty could not be solved by smoothing the point-like interaction by a massive, and therefore short range, charged vector particle exchange (the so-called $W^{+}$and $W^{-}$mesons); theories with fundamental massive charged vector mesons are not renormalizable either. In the early nineteen sixties, there seemed to be insuperable obstacles for formulating a theory with short range forces mediated by massive vectors.

The solution of the latter problem came from the theory proposed in 1964 by Brout and Englert [四] and by Higgs [2, 目]. The Brout-Englert-Higgs (BEH) theory is based on a mechanism, inspired from the spontaneous symmetry breaking of a continuous symmetry, discussed in the previous talk by Robert Brout, adapted to gauge theories and in particular to non abelian gauge theories. The mechanism unifies long range and short range forces mediated by vector mesons, by deriving the vector mesons masses from a fundamental theory containing only massless vector fields. It led to a solution of the weak interaction puzzle and opened the way to modern perspectives on unified laws of nature.

Before turning to an exposé of the BEH mechanism, we shall in section II review, in the context of quantum field theory, the analysis given by Robert Brout of the spontaneous breaking of a continuous symmetry. Section III explains the BEH mechanism. We present the quantum field theory approach of Brout and Englert wherein the breaking mechanism for both abelian and non abelian gauge groups is induced by scalar bosons. We also present their approach in the case of dynamical symmetry breaking from fermion condensate. We then turn to the equation of motion approach of Higgs. Finally we explain the renormalization issue. In section IV, we briefly review the well-known applications of the BEH mechanism with particular emphasis on concepts relevant to the quest for unification. Some comments on this subject are made in section V.

\section{Spontaneous Breaking of a Global Symmetry}

Spontaneous breaking of a Lie group symmetry was discussed by Robert Brout in "The Paleolitic Age". I review here its essential features in the quantum field theory context. 
Recall that spontaneous breakdown of a continuous symmetry in condensed matter physics implies a degeneracy of the ground state, and as a consequence, in absence of long range forces, collective modes appear whose energies go to zero when the wavelength goes to infinity. This was exemplified in particular by spin waves in a Heisenberg ferromagnet. There, the broken symmetry is the rotation invariance.

Spontaneous symmetry breaking was introduced in relativistic quantum field theory by Nambu in analogy to the BCS theory of superconductivity. The problem studied by Nambu [四] and Nambu and Jona-Lasinio [5] is the spontaneous breaking of chiral symmetry induced by a fermion condensate $^{1}$. The chiral phase group $\exp \left(i \gamma_{5} \alpha\right)$ is broken by the fermion condensate $\langle\bar{\psi} \psi\rangle \neq 0$ and the massless mode is identified with the pion. The latter gets its tiny mass (on the hadron scale) from a small explicit breaking of the symmetry, just as a small external magnetic field imparts a small gap in the spin wave spectrum. This interpretation of the pion mass constituted a breakthrough in our understanding of strong interaction physics. General features of spontaneous symmetry breakdown in relativistic quantum field theory were further formalized by Goldstone [ 6 ]. Here, symmetry is broken by non vanishing vacuum expectation values of scalar fields. The method is designed to exhibit the appearance of a massless mode out of the degenerate vacuum and does not really depend on the significance of the scalar fields. The latter could be elementary or represent collective variables of more fundamental fields, as would be the case in the original Nambu model. Compositeness affects details of the model considered, such as the behavior at high momentum transfer, but not the existence of the massless excitations encoded in the degeneracy of the vacuum.

Let us first illustrate the occurrence of this massless Nambu-Goldstone (NG) boson in a simple model of a complex scalar field with $U(1)$ symmetry [ [6].

The Lagrangian density,

$$
\mathscr{L}=\partial^{\mu} \phi^{*} \partial_{\mu} \phi-V\left(\phi^{*} \phi\right) \quad \text { with } \quad V\left(\phi^{*} \phi\right)=-\mu^{2} \phi^{*} \phi+\lambda\left(\phi^{*} \phi\right)^{2}, \lambda>0,
$$

is invariant under the $U(1)$ group $\phi \rightarrow e^{i \alpha} \phi$. The $U(1)$ symmetry is called global because the group parameter $\alpha$ is constant in space-time. It is broken by a vacuum expectation value of the $\phi$-field given, at the classical level, by the minimum of $V\left(\phi^{*} \phi\right)$. Writing $\phi=\left(\phi_{1}+i \phi_{2}\right) / \sqrt{2}$, one may choose $\left\langle\phi_{2}\right\rangle=0$. Hence $\left\langle\phi_{1}\right\rangle^{2}=\mu^{2} / \lambda$ and we select, say, the vacuum with $\left\langle\phi_{1}\right\rangle$ positive. The potential $V\left(\phi^{*} \phi\right)$ is depicted in Fig.1 .

Around the unbroken vacuum the field $\phi_{1}$ has negative mass and acquires a positive mass around the broken vacuum where the field $\phi_{2}$ is massless. The latter is the NG boson of broken $\mathrm{U}(1)$ symmetry. The massive scalar describes the fluctuations of the order parameter $\left\langle\phi_{1}\right\rangle$. Its mass is the analog of the inverse longitudinal susceptibility of the Heisenberg ferromagnet discussed by Robert Brout while the vanishing of the NG boson mass corresponds to the vanishing of its inverse transverse susceptibility. The scalar boson $\phi_{1}$ is always present in spontaneous breakdown of a symmetry. In the context of the BEH mechanism analyzed in the following section, it was introduced by Brout and myself, and by Higgs. We shall label it the BEH boson ${ }^{2}$ (Fig.1).

In the classical limit, the origin of the massless NG boson $\phi_{2}$ is clearly illustrated in the Fig.1. The vacuum characterized by the order parameter $\left\langle\phi_{1}\right\rangle$ is rotated into an equivalent vacuum by the

\footnotetext{
${ }^{1}$ See the detailed discussion in Brout's lecture, section VII.

${ }^{2}$ It is often called the Higgs boson in the literature.
} 


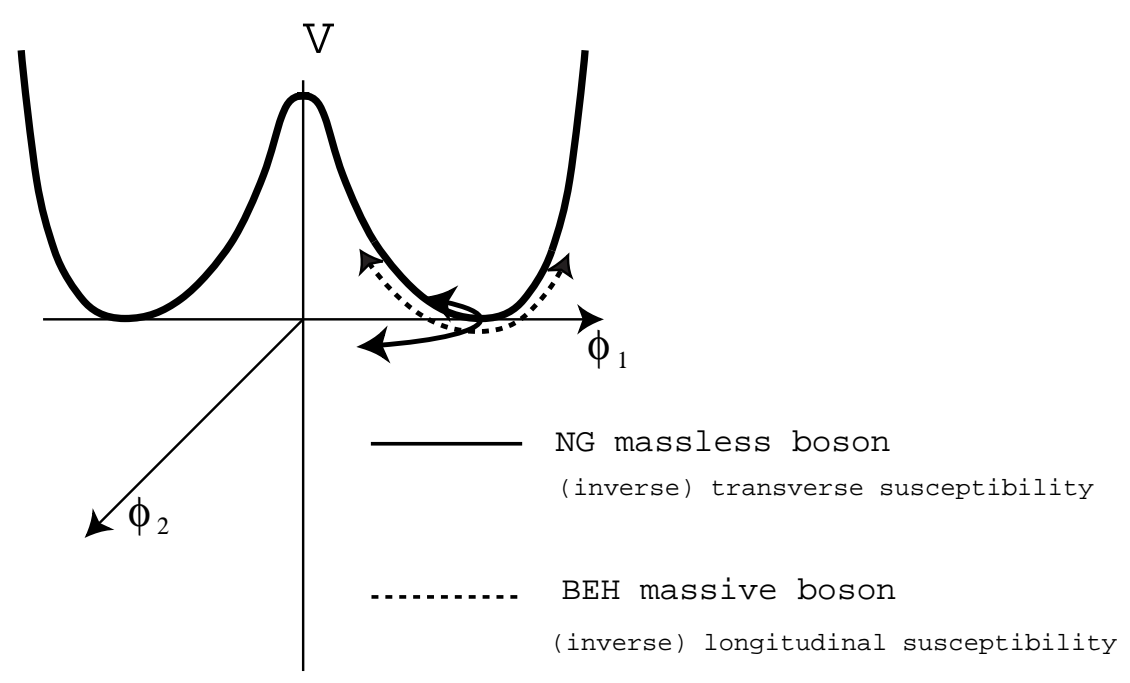

Fig. 1

field $\phi_{2}$ at zero space momentum. Such rotation costs no energy and thus the field $\phi_{2}$ at space momenta $\vec{q}=0$ has $q_{0}=0$ on the equations of motion, and hence zero mass.

This can be formalized and generalized by noting that the conserved Noether current $J_{\mu}=$ $\phi_{1} \partial_{\mu} \phi_{2}-\phi_{2} \partial_{\mu} \phi_{1}$ gives a charge $Q=\int J_{0} d^{3} x$. The operator $\exp (i \alpha Q)$ rotates the vacuum by an angle $\alpha$. In the classical limit, this charge is, around the chosen vacuum, $Q=\int\left\langle\phi_{1}\right\rangle \partial_{0} \phi_{2} d^{3} x$ and involves only $\phi_{2}$ at zero momentum. In general, $\left\langle\left[Q, \phi_{2}\right]\right\rangle=i\left\langle\phi_{1}\right\rangle$ is non zero in the chosen vacuum. This implies that the propagator $\partial^{\mu}\left\langle T J_{\mu}(x) \phi_{2}\left(x^{\prime}\right)\right\rangle$ cannot vanish at zero four-momentum $q$ because its integral over space-time is precisely $\left\langle\left[Q, \phi_{2}\right]\right\rangle$. Expressing the propagator in terms of Feynman diagrams we see that the $\phi_{2}$-propagator must have a pole at $q^{2}=0$. The field $\phi_{2}$ is the massless NG boson.

The proof is immediately extended to the spontaneous breaking of a semi-simple Lie group global symmetry. Let $\phi^{A}$ be scalar fields spanning a representation of the Lie group $\mathscr{G}$ generated by the (antihermitian) matrices $T^{a A B}$. If the dynamics is governed by a $\mathscr{G}$-invariant action and if the potential has minima for non vanishing $\phi^{A}, \mathrm{~s}$, symmetry is broken and the vacuum is degenerate under $\mathscr{G}$-rotations. The conserved charges are $Q^{a}=\int \partial_{\mu} \phi^{B} T^{a B A} \phi^{A} d^{3} x$. As in the abelian case above, the propagators of the fields $\phi^{B}$ such that $\left\langle\left[Q^{a}, \phi^{B}\right]\right\rangle=T^{a B A}\left\langle\phi^{A}\right\rangle \neq 0$ have a NG pole at $q^{2}=0$.

\section{The BEH Mechanism}

\subsection{From global to local symmetry}

The global $U(1)$ symmetry in Eq.([2. $e^{i \alpha(x)} \phi(x)$ by introducing a vector field $A_{\mu}(x)$ transforming according to $A_{\mu}(x) \rightarrow A_{\mu}(x)+(1 / e) \partial_{\mu} \alpha(x)$. The corresponding Lagrangian density is

$$
\mathscr{L}=D^{\mu} \phi^{*} D_{\mu} \phi-V\left(\phi^{*} \phi\right)-\frac{1}{4} F_{\mu v} F^{\mu v}
$$


with covariant derivative $D_{\mu} \phi=\partial_{\mu} \phi-i e A_{\mu} \phi$ and $F_{\mu \nu}=\partial_{\mu} A_{v}-\partial_{v} A_{\mu}$.

Local invariance under a semi-simple Lie group $\mathscr{G}$ can be realized by extending the Lagrangian Eq.(B. (I) to incorporate non-abelian Yang-Mills vector fields $A_{\mu}^{a}$

$$
\mathscr{L}_{\mathscr{G}}=\left(D^{\mu} \phi\right)^{* A}\left(D_{\mu} \phi\right)^{A}-V-\frac{1}{4} F_{\mu v}^{a} F^{a \mu v},
$$

where

$$
\left(D_{\mu} \phi\right)^{A}=\partial_{\mu} \phi^{A}-e A_{\mu}^{a} T^{a A B} \phi^{B}, F_{\mu \nu}^{a}=\partial_{\mu} A_{v}^{a}-\partial_{v} A_{\mu}^{a}-e f^{a b c} A_{\mu}^{b} A_{v}^{c} .
$$

Here, $\phi^{A}$ belongs to the representation of $\mathscr{G}$ generated by $T^{a A B}$ and the potential $V$ is invariant under $\mathscr{G}$.

The success of quantum electrodynamics based on local U(1) symmetry, and of classical general relativity based on a local generalization of Poincare invariance, provides ample evidence for the relevance of local symmetry for the description of natural laws. One expects that local symmetry has a fundamental significance rooted in causality and in the existence of exact conservation laws at a fundamental level, of which charge conservation appears as the prototype. As an example of the strength of local symmetry we cite the fact that conservation laws resulting from a global symmetry alone are violated in presence of black holes.

The local symmetry, or gauge invariance, of Yang-Mills theory, abelian or non abelian, apparently relies on the massless character of the gauge fields $A_{\mu}$, hence on the long range character of the forces they transmit, as the addition of a mass term for $A_{\mu}$ in the Lagrangian Eq.(B.D) or (B.2.) destroys gauge invariance. But short range forces, such as the weak interaction forces, seem to be as fundamental as the electromagnetic ones despite the apparent absence of exact conservation laws. To reach a basic description of such forces one is tempted to link the violation of conservation to a mass of the gauge fields which would arise from spontaneous symmetry breaking. However the problem of spontaneous broken symmetry is different for global and for local symmetry.

To understand the difference, let us break the symmetries explicitly. To the Lagrangian Eq.(2.1) we add the term

$$
\phi h^{*}+\phi^{*} h,
$$

where $h, h^{*}$ are constant in space time. Let us take $\mathrm{h}$ real. The presence of the field $h$ breaks explicitly the global $U(1)$ symmetry and the field $\phi_{1}$ always develops an expectation value. When $h \rightarrow 0$, the symmetry of the action is restored but, when the symmetry is broken by a minimum of $V\left(\phi \phi^{*}\right)$ at $|\phi| \neq 0$, we still have $\left\langle\phi_{1}\right\rangle \neq 0$. The tiny $h$-field simply picks up one of the degenerate vacua in perfect analogy with the infinitesimal magnetic field which orients the magnetization of a ferromagnet. As in statistical mechanics, spontaneous broken global symmetry can be recovered in the limit of vanishing external symmetry breaking. The degeneracy of the vacuum can be put into evidence by changing the phase of $h$; in this way, we can reach in the limit $h \rightarrow 0$ any $U(1)$ rotated vacuum.

When the symmetry is extended from global to local, one can still break the symmetry by an external "magnetic" field. However in the limit of vanishing magnetic field the expectation value of any gauge dependent local operator will tend to zero because, in contradistinction to global symmetry, it cost no energy in the limit to change the relative orientation of neighboring "spins"; there is then no ordered configuration in group space which can be protected from disordering fluctuations. 


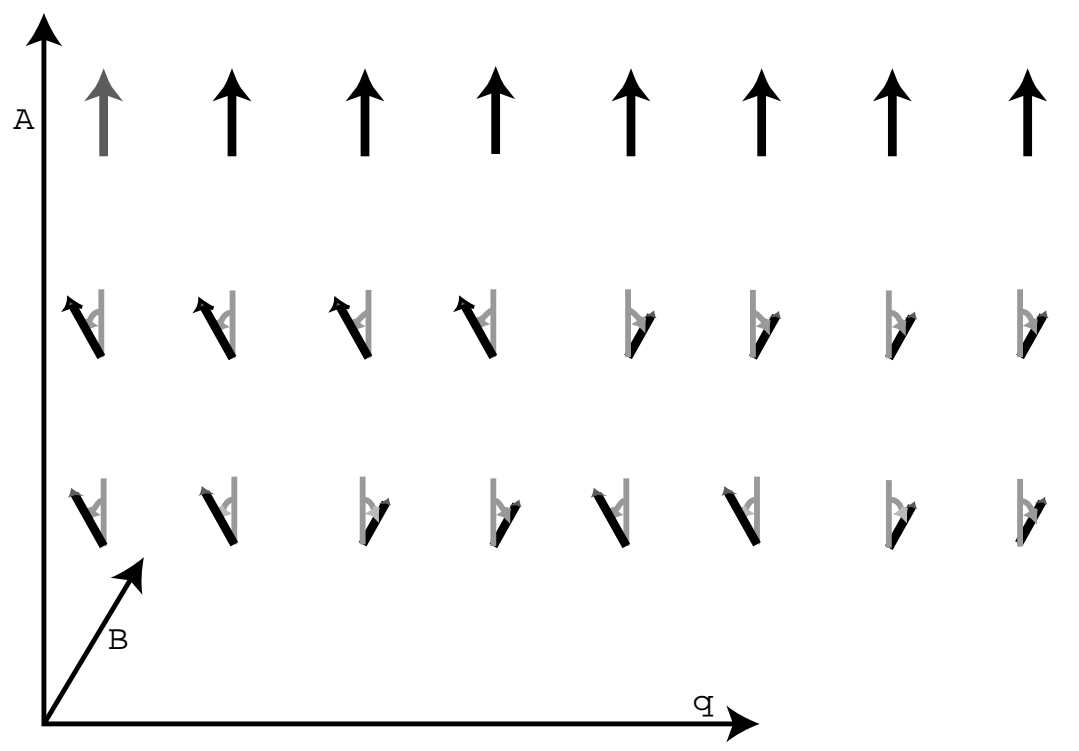

Fig. 2

As a consequence, the vacuum is generically non degenerate and points in no particular direction in group space as the external field goes to zero. Local gauge symmetry cannot be spontaneously broken $^{3}$ and the vacuum is gauge invariant ${ }^{4}$. Recalling that the explicit presence of a gauge vector mass breaks gauge invariance, we are thus faced with a dilemma. How can gauge fields acquire mass without breaking the local symmetry?

\subsection{Solving the dilemma}

In perturbation theory, gauge invariant quantities are evaluated by choosing a particular gauge. One imposes the gauge condition by adding to the action a gauge fixing term and one sums over subsets of graphs satisfying the Ward Identities ${ }^{5}$.

Consider the Yang-Mills theory defined by the Lagrangian Eq.(B.2). Let us choose a gauge which preserves Lorentz invariance and a residual global $\mathscr{G}$ symmetry. This can be achieved by adding to the Lagrangian a gauge fixing term $(2 \eta)^{-1} \partial_{\mu} A_{a}^{\mu} \partial_{\nu} A^{a \nu}$. The gauge parameter $\eta$ is arbitrary and has no observable consequences.

The global symmetry can now be spontaneously broken, for suitable potential $V$, by non zero expectation values $\left\langle\phi^{A}\right\rangle$ of BEH fields. In Fig.2 we have represented fluctuations of this parameter in the spatial $q$-direction and in an internal space direction orthogonal to the direction $A$. The orthogonal direction depicted in the figure has been labeled $B$. Fig.2a pictures the spontaneously

\footnotetext{
${ }^{3}$ For a detailed proof, see reference [8]].

${ }^{4}$ Note that for global symmetry breaking, one can always choose a linear combination of degenerate vacua which is invariant under, say, the $U(1)$ symmetry. This choice has no observable consequences and only masks the degeneracy of the vacuum which is guaranteed by a superselection rule. The Hilbert space splits indeed, as in the ferromagnetic case analyzed by Robert Brout (section V of "The Paleolitic Age"), into an infinite number of orthogonal spaces formed by all the finite excitations on each degenerate vacuum.

${ }^{5}$ To this end, it is often necessary, in particular for non abelian gauge theories, to include Fadeev-Popov ghosts terms in the action. These contribute when closed gauge field loops are included in the computation.
} 

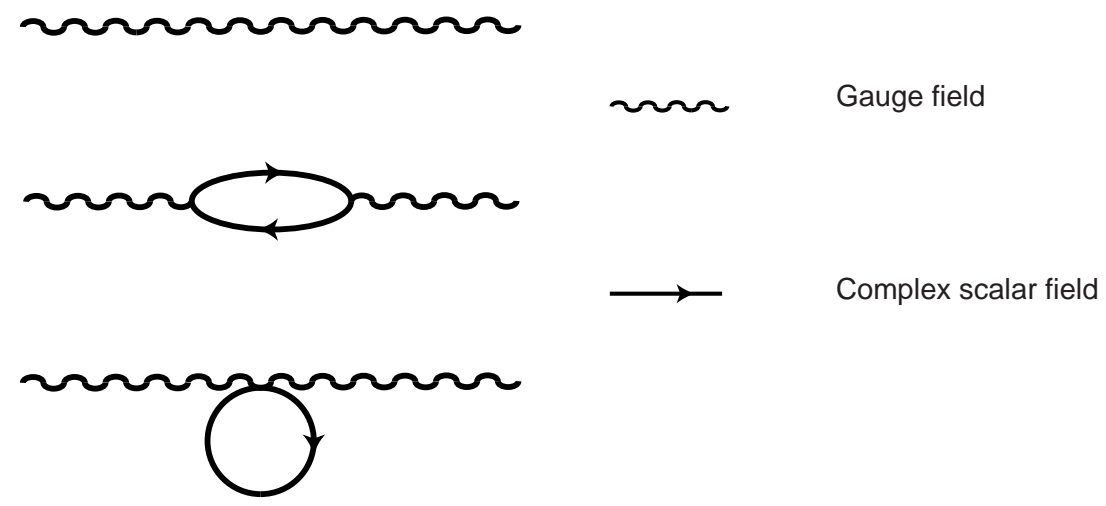

Fig. 3

broken vacuum of the gauge fixed Lagrangian. Fig.2b and $2 \mathrm{c}$ represent fluctuations of finite wavelength $\lambda$.

Clearly as $\lambda \rightarrow \infty$ these fluctuations can only induce global rotations in the internal space. In absence of gauge fields, such fluctuations would give rise, as in spontaneously broken global continuous symmetries, to massless NG mode. In a gauge theory, fluctuations of $\left\langle\phi^{A}\right\rangle$ are just local rotations in the internal space and hence are unobservable gauge fluctuations. Hence the NG bosons induce only gauge transformations and its excitations disappear from the physical spectrum.

The degrees of freedom of the NG fields were present in the original gauge invariant action and cannot disappear. But what makes local internal space rotations unobservable in a gauge theory is precisely the fact that they can be absorbed through gauge transformations by the Yang-Mills fields. The absorption of the long range NG fields renders massive those gauge fields to which they are coupled, and transfers to them the missing degrees of freedom which becomes their third polarization.

We shall see in the next sections how these considerations are realized in quantum field theory, giving rise to an apparent breakdown of symmetry: despite the absence of spontaneous local symmetry breaking, gauge invariant vector masses will be generated in a coset $\mathscr{G} / \mathscr{H}$, leaving long range forces only in a subgroup $\mathscr{H}$ of $\mathscr{G}$.

\subsection{The quantum field theory approach [U]}

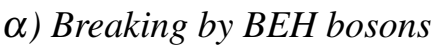

Let us first examine the abelian case as realized by the complex scalar field $\phi$ exemplified in Eq.(B.J).

In the covariant gauges, the free propagator of the field $A_{\mu}$ is

$$
D_{\mu v}^{0}=\frac{g_{\mu v}-q_{\mu} q_{v} / q^{2}}{q^{2}}+\eta \frac{q_{\mu} q_{v} / q^{2}}{q^{2}}
$$

where $\eta$ is the gauge parameter. It can be put equal to zero, as in the Landau gauge used in reference [四], but we leave it arbitrary here to illustrate explicitly the role of the NG-boson.

In absence of symmetry breaking, the lowest order contribution to the self-energy, arising from the covariant derivative terms in Eq.(B.D), is given by the one-loop diagrams of Fig.3. The 


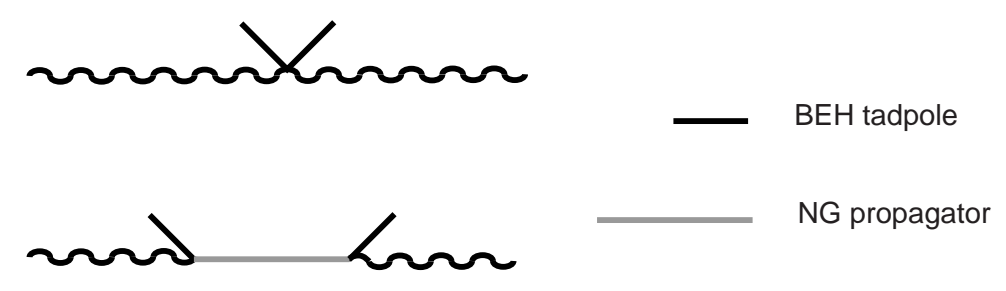

Fig. 4

self-energy (suitably regularized) takes the form of a polarization tensor

$$
\Pi_{\mu v}=\left(g_{\mu v} q^{2}-q_{\mu} q_{v}\right) \Pi\left(q^{2}\right),
$$

where the scalar polarisation $\Pi\left(q^{2}\right)$ is regular at $q^{2}=0$, leading to the gauge field propagator

$$
D_{\mu v}=\frac{g_{\mu v}-q_{\mu} q_{v} / q^{2}}{q^{2}\left[1-\Pi\left(q^{2}\right)\right]}+\eta \frac{q_{\mu} q_{v} / q^{2}}{q^{2}}
$$

The polarization tensor in Eq.(B.6) is transverse and hence does not affect the gauge parameter $\eta$. The transversality of the polarization tensor reflects the gauge invariance of the theory ${ }^{6}$ and, as we shall see below, the regularity of the polarization scalar signals the absence of symmetry breaking. This guarantees that the $A_{\mu}$-field remains massless.

Symmetry breaking adds tadpole diagrams to the previous ones. To see this write

$$
\phi=\frac{1}{\sqrt{2}}\left(\phi_{1}+i \phi_{2}\right) \quad\left\langle\phi_{1}\right\rangle \neq 0 .
$$

The BEH field is $\phi_{1}$ and the NG field $\phi_{2}$. The additional diagrams are depicted in Fig.4. In this case, the polarisation scalar $\Pi\left(q^{2}\right)$ in Eq.(B., (B) acquires a pole

$$
\Pi\left(q^{2}\right)=\frac{e^{2}\left\langle\phi_{1}\right\rangle^{2}}{q^{2}}
$$

and, in lowest order perturbation theory, the gauge field propagator becomes

$$
D_{\mu v}=\frac{g_{\mu v}-q_{\mu} q_{v} / q^{2}}{q^{2}-\mu^{2}}+\eta \frac{q_{\mu} q_{v} / q^{2}}{q^{2}}
$$

which shows that the $A_{\mu}$-field gets a mass

$$
\mu^{2}=e^{2}\left\langle\phi_{1}\right\rangle^{2} .
$$

The generalization of Eqs.(B.6) and (B.9) to the non abelian case described by the action Eq.(B.2) is straightforward. One gets from the graphs depicted in Fig.5,

$$
\begin{aligned}
& \Pi_{\mu \nu}^{a b}=\left(g_{\mu \nu} q^{2}-q_{\mu} q_{v}\right) \Pi^{a b}\left(q^{2}\right), \\
& \Pi^{a b}\left(q^{2}\right)=\frac{e^{2}\left\langle\phi^{* B}\right\rangle T^{* a B C} T^{b C A}\left\langle\phi^{A}\right\rangle}{q^{2}},
\end{aligned}
$$

\footnotetext{
${ }^{6}$ The transversality of polarisation tensors is a consequence of the Ward Identities alluded to in the preceding section.
} 


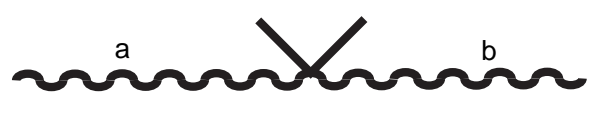

Fig. 5

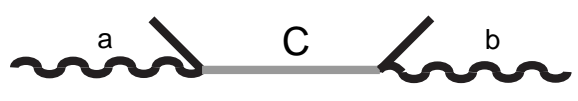

from which follows the mass matrix

$$
\mu^{a b}=e^{2}\left\langle\phi^{* B}\right\rangle T^{* a B C} T^{b C A}\left\langle\phi^{A}\right\rangle
$$

In terms of the non-zero eigenvalues $\mu_{a}$ of the mass matrix the propagator for the massive gauge vectors takes the same form as Eq.(B.JU)

$$
D_{\mu v}^{a}=\frac{g_{\mu v}-q_{\mu} q_{v} / q^{2}}{q^{2}-\mu^{a 2}}+\eta \frac{q_{\mu} q_{v} / q^{2}}{q^{2}}
$$

The gauge invariance is expressed, as it was in absence of symmetry breaking, through the transversality of the polarization tensors Eqs.(B.6) and (B.12). The singular $1 / q^{2}$ contributions to the polarization scalars Eqs.(B.9) and (B..3), which preserve transversality while giving mass to the gauge fields, stem from the long range NG boson fields encoded in their $1 / q^{2}$ propagator. We shall verify below that this pole has no observable effect as such. On the other hand, its absorption in the gauge field propagator transfers the degrees of freedom of the NG bosons to the third degree of polarization of the massive vectors. Indeed, on the mass shell $q^{2}=\mu^{a 2}$, one easily verifies that the numerator in their propagator Eq.(B.5) is:

$$
g_{\mu v}-\frac{q_{\mu} q_{v}}{q^{2}}=\sum_{\lambda=1}^{3} e_{\mu}^{(\lambda)} \cdot e_{v}^{(\lambda)}, q^{2}=\mu^{a 2},
$$

where the $e_{\mu}^{(\lambda)}$ are the three polarization vectors which are orthonormal in the rest frame of the particle.

In this way, the NG bosons generate massive propagators for those gauge fields to which they are coupled. Long range forces only survive in the subgroup $\mathscr{H}$ of $\mathscr{G}$ which leaves invariant the non vanishing expectation values $\left\langle\phi^{A}\right\rangle$.

Note that (as in the abelian case) the scalar potential $V$ does not enter the computation of the gauge field propagator. This is because the trilinear term arising from the covariant derivatives in the Lagrangian Eq.(B.2), which yields the second graph of Fig.5, can only couple the tadpoles to other scalar fields through group rotations and hence couple them only to the NG bosons. These are the eigenvectors with zero eigenvalue of the scalar mass matrix given by the quadratic term in the expansion of the potential $V$ around its minimum. Hence the mass matrix decouples from the tadpole at the tree level considered above. An explicit example of this feature will be given for the Lagrangian Eq.(4.5).

$\beta$ ) Dynamical symmetry breaking 


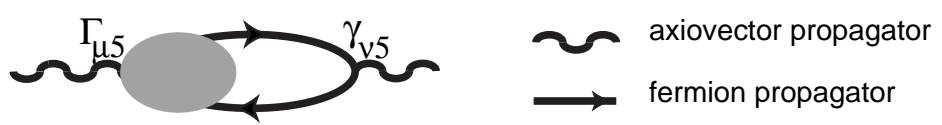

Fig.6

The symmetry breaking giving mass to gauge vector bosons may arise from the fermion condensate breaking chiral symmetry. This is illustrated by the following chiral invariant Lagrangian

$$
\mathscr{L}=\mathscr{L}_{0}^{F}-e_{V} \bar{\psi} \gamma_{\mu} \psi V_{\mu}-e_{A} \bar{\psi} \gamma_{\mu} \gamma_{5} \psi A_{\mu}-\frac{1}{4} F_{\mu v} F^{\mu v}(V)-\frac{1}{4} F_{\mu v} F^{\mu v}(A)
$$

Here $F_{\mu v}(V)$ and $F_{\mu v}(A)$ are abelian field strength for $U(1) \times U(1)$ symmetry. Chiral anomalies are eventually canceled by adding in the required additional fermions.

The Ward identity for the chiral current

$$
q^{\mu} \Gamma_{\mu 5}(p+q / 2, p-q / 2)=S^{-1}(p+q / 2) \gamma_{5}+\gamma_{5} S^{-1}(p-q / 2),
$$

shows that if the fermion self-energy $\gamma^{\mu} p_{\mu} \Sigma_{2}\left(p^{2}\right)-\Sigma_{1}\left(p^{2}\right)$ acquires a non vanishing $\Sigma_{1}\left(p^{2}\right)$ term, thus a dynamical mass $m$ at $\Sigma_{1}\left(m^{2}\right)=m$ (taking for simplicity $\Sigma_{2}\left(m^{2}\right)=1$ ), the axial vertex $\Gamma_{\mu 5}$ develops a pole at $q^{2}=0$. In leading order in $q$, we get

$$
\Gamma_{\mu 5} \rightarrow 2 m \gamma_{5} \frac{q_{\mu}}{q^{2}}
$$

The pole in the vertex function induces a pole in the suitably regularized gauge invariant polarization tensor $\Pi_{\mu \nu}^{(A)}$ of the axial vector field $A_{\mu}$ depicted in Fig.6

$$
\Pi_{\mu \nu}^{(A)}=e_{A}^{2}\left(g_{\mu \nu} q^{2}-q_{\mu} q_{v}\right) \Pi^{(A)}\left(q^{2}\right)
$$

with

$$
\lim _{q^{2} \rightarrow 0} q^{2} \Pi^{(A)}\left(q^{2}\right)=\mu^{2} \neq 0
$$

The field $A_{\mu}$ acquires in this approximation ${ }^{7}$ a gauge invariant mass $\mu$.

This example illustrates the fact that the transversality of the polarization tensor used in the quantum field theoretic approach to mass generation is a consequence of a Ward identity. This is true whether vector masses arise through fundamental fundamental BEH bosons or through fermion condensate. The generation of gauge invariant masses is therefore not contingent upon the "tree

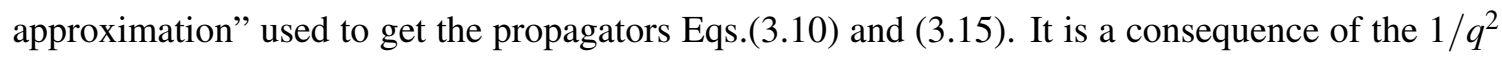
singularity in the vacuum polarisation scalars Eqs.(B.9), (B.12) or (B.2] ) which comes from NG boson contribution.

\footnotetext{
${ }^{7}$ The validity of the approximation, and in fact of the dynamical approach, rests on the high momentum behavior of the fermion self energy, but this problem will not be discussed here.
} 


\subsection{The equation of motion approach [2], [3]}

Shortly after the above analysis was presented, Higgs wrote two papers. In the first one [i] , he showed that the proof of the Goldstone theorem [ [6, U], which states that, in relativistic quantum field theory, spontaneous symmetry breaking of a continuous global symmetry implies zero mass NG bosons, fails in the case of gauge field theory. In the second paper [ []] , he derived the BEH theory in terms of the classical equations of motion, which he formulated for the abelian case.

From the action Eq.(B.]), taking as in Eq.(B.8), the expectation value of the BEH boson to be $\left\langle\phi_{1}\right\rangle$, and expanding the NG field $\phi_{2}$ to first order, one gets the classical equations of motion to that order

$$
\begin{aligned}
& \partial^{\mu}\left\{\partial_{\mu} \phi_{2}-e\left\langle\phi_{1}\right\rangle A_{\mu}\right\}=0, \\
& \partial_{v} F^{\mu v}=e\left\langle\phi_{1}\right\rangle\left\{\partial^{\mu} \phi_{2}-e\left\langle\phi_{1}\right\rangle A^{\mu}\right\} .
\end{aligned}
$$

Defining

$$
B_{\mu}=A_{\mu}-\frac{1}{e\left\langle\phi_{1}\right\rangle} \partial_{\mu} \phi_{2} \text { and } G_{\mu v}=\partial_{\mu} B_{v}-\partial_{v} B_{\mu}=F_{\mu \nu}
$$

one gets

$$
\partial_{\mu} B^{\mu}=0, \quad \partial_{v} G^{\mu v}+e^{2}\left\langle\phi_{1}\right\rangle^{2} B^{\mu}=0 .
$$

Eq.(B.25) shows that $B_{\mu}$ is a massive vector field with mass squared $e^{2}\left\langle\phi_{1}\right\rangle^{2}$ in accordance with Eq.(B.II).

In this formulation, we see clearly how the Goldstone boson is absorbed into a redefined massive vector field which has no longer explicit gauge invariance. The same phenomenon in the quantum field theory approach is related to the unobservability of the $1 / q^{2}$ pole mentioned in the discussion of Eq.(B.14); this will be made explicit in the next section.

The equation of motion approach is classical in character but, as pointed out by Higgs [B]], the formulation of the BEH mechanism in the quantum field theory terms of reference [四] indicates its validity in the quantum regime. We now show how the latter formulation signals the renormalizability of the BEH theory.

\subsection{The renormalization issue}

The massive vector propagator Eq.(B.15) differs from a conventional free massive propagator in two respects. First the presence of the unobservable longitudinal term reflects the arbitrariness of the gauge parameter $\eta$. Second the NG pole at $q^{2}=0$ in the transverse projector $g_{\mu \nu}-q_{\mu} q_{v} / q^{2}$ is unconventional. Its significance is made clear by expressing the propagator of the $A_{\mu}$ field in Eq.(3.15) as (putting $\eta$ to zero)

$$
D_{\mu \nu}^{a} \equiv \frac{g_{\mu \nu}-q_{\mu} q_{v} / q^{2}}{q^{2}-\mu^{a 2}}=\frac{g_{\mu v}-q_{\mu} q_{v} / \mu^{a 2}}{q^{2}-\mu^{a 2}}+\frac{1}{\mu^{a 2}} \frac{q_{\mu} q_{v}}{q^{2}} .
$$

The first term in the right hand side of Eq.(B.26) is the conventional massive vector propagator. It may be viewed as the (non-abelian generalization of the) free propagator of the $B_{\mu}$ field defined in Eq.(B.24) while the second term is a pure gauge propagator due to the NG boson $\left(\left[1 / e\left\langle\phi_{1}\right\rangle\right] \partial_{\mu} \phi_{2}\right.$ in Eq.(B.24) ) which converts the $A_{\mu}$ field into this massive vector field $B_{\mu}$. 
The propagator Eq.(B.15) which appeared in the field theoretic approach contains thus, in the covariant gauges, the transverse projector $g_{\mu \nu}-q_{\mu} q_{v} / q^{2}$ in the numerator of the massive gauge field $A_{\mu}^{a}$ propagator. This is in sharp contradistinction to the numerator $g_{\mu v}-q_{\mu} q_{v} / \mu^{a 2}$ characteristic of the conventional massive vector field $B_{\mu}$ propagator. It is the transversality of the self energy in covariant gauges, which led in the "tree approximation" to the transverse projector in Eq.(3.55). As already mentioned, the transversality is a consequence of a Ward identity and therefore does not depend on the tree approximation. This fact is already suggested from the dynamical example presented above but was proven in more general terms in a subsequent publication ${ }^{8}[Q]$. The importance of this fact is that the transversality of the self-energy in covariant gauges determines the power counting of irreducible diagrams. It is then straightforward to verify that the BEH quantum field theory formulation is renormalizable by power counting.

On this basis we suggested that the BEH theory constitutes indeed a consistent renormalizable field theory [Q]. To prove this statement, one must verify that the theory is unitary, a fact which is not apparent in the "renormalizable" covariant gauges because of the $1 / q^{2}$ pole in the projector, but would be manifest in the "unitary gauge" defined in the free theory by the $B_{\mu}$ propagator. In the unitary gauge however, renormalization from power counting is not manifest. The equivalence, at the free level, between the $A_{\mu}$ and $B_{\mu}$ free propagators, which is only true in a gauge invariant theory where their difference is the unobservable NG propagator appearing in Eq.(13.26), is the clue of the consistency of the BEH theory. A full proof that the theory is renormalizable and unitary was achieved by 't Hooft and Veltman [ए]]].

\section{Consequences}

The most dramatic application of the BEH mechanism is the electroweak theory, amply confirmed by experiment. Considerable work has been done, using the BEH mechanism, to formulate Grand Unified theories of non gravitational interactions. We shall summarize here these well known ideas and then evoke the construction of regular monopoles and flux lines using BEH bosons, because they raise potentially important conceptual issues. We shall also mention briefly the attempts to include gravity in the unification quest, in the so called M-theory approach, and focuses in this context on an interesting geometrical interpretation of the BEH mechanism.

\subsection{The electroweak theory [i]]}

In the electroweak theory, the gauge group is taken to be $S U(2) \times U(1)$ with corresponding generators and coupling constants $g A_{\mu}^{a} T^{a}$ and $g^{\prime} B_{\mu} Y^{\prime}$. The $S U(2)$ acts on left-handed fermions only. The electromagnetic charge operator is $Q=T^{3}+Y^{\prime}$ and the electric charge $e$ is usually expressed in terms of the mixing angle $\theta$ as $g=e / \sin \theta, g^{\prime}=e / \cos \theta$. The BEH bosons $\left(\phi^{+}, \phi^{0}\right)$ are in a doublet of $S U(2)$ and their $U(1)$ charge is $Y^{\prime}=1 / 2$. Breaking occurs in such a way that $Q$ generates an unbroken subgroup, coupled to which is the massless photon field. Thus the vacuum is characterized by $\langle\phi\rangle=1 / \sqrt{2}(0, v)$.

Using Eqs.(B.TI) and (B.T4) we get the mass matrix

\footnotetext{
${ }^{8}$ The proof given in reference [ $[$ ] was not complete because closed Yang-Mills loops, which would have required the introduction of Fadeev-Popov ghosts were not included.
} 


$$
\left|\mu^{2}\right|=\frac{v^{2}}{4}\left|\begin{array}{cccc}
g^{2} & 0 & 0 & 0 \\
0 & g^{2} & 0 & 0 \\
0 & 0 & g^{\prime 2} & -g g^{\prime} \\
0 & 0 & -g g^{\prime} & g^{2}
\end{array}\right|
$$

whose diagonalization yields the eigenvalues

$$
M_{W^{+}}^{2}=\frac{v^{2}}{4} g^{2}, M_{W^{-}}^{2}=\frac{v^{2}}{4} g^{2}, M_{Z}^{2}=\frac{v^{2}}{4}\left(g^{\prime 2}+g^{2}\right), M_{A}^{2}=0 .
$$

This permits to relate $v$ to the the Fermi coupling $G$ as $v^{2}=(\sqrt{2} G)^{-1}$.

Although the electroweak theory has been amply verified by experiment, the existence of the BEH boson has, as yet, not been confirmed. It should be noted that the physics of the BEH boson is more sensitive to dynamical assumptions than the massive vectors $W^{ \pm}$and $Z$, be it a genuine elementary field or a manifestation of a composite due to a more elaborate mechanism. Hence observation of its mass and width is of particular interest for further understanding of the mechanism at work.

\subsection{Grand unification schemes}

The discovery that confinement could be explained by the strong coupling limit of quantum chromodynamics based on the "color" gauge group $S U(3)$ led to tentative Grand Unification schemes where electroweak and strong interaction could be unified in a simple gauge group $\mathscr{G}$ containing $S U(2) \times U(1) \times S U(3)$ [ए]]. Breaking occurs through vacuum expectation values of BEH fields and unification can be realized at high energies because while the renormalization group makes the small gauge coupling of $U(1)$ increase logarithmically with the energy scale, the converse is true for the asymptotically free non abelian gauge groups.

\subsection{Monopoles, flux tubes and electromagnetic duality}

In electromagnetism, monopoles can be included at the expense of introducing a Dirac string [113]. The latter creates a singular potential along the string terminating at the monopole. For instance to describe a point-like monopole located at $\vec{r}=0$, one can take the line-singular potential

$$
\vec{A}=\frac{g}{4 \pi}(1-\cos \theta) \vec{\nabla} \phi
$$

This potential has a singularity along the negative $z$-axis $(\theta=\pi)$ where the string has been put (see Fig.7). The unobservability of the string implies that its fictitious flux be quantized according to the Dirac condition

$$
e g=2 \pi n \quad n \in Z
$$

In contradistinction to the string in the $U(1)$ theory, the Dirac string in non abelian gauge groups can be removed by a gauge singularity for well chosen quantized magnetic charges, reducing the line singularity to a point like singularity.

An example is the $\mathrm{SO}(3)$ monopole, represented in Fig.8, arising from the potential

$$
A^{a i}=\frac{g}{4 \pi} \varepsilon^{i a b} \frac{r^{b}}{r^{2}} \quad, \quad e g=4 \pi
$$



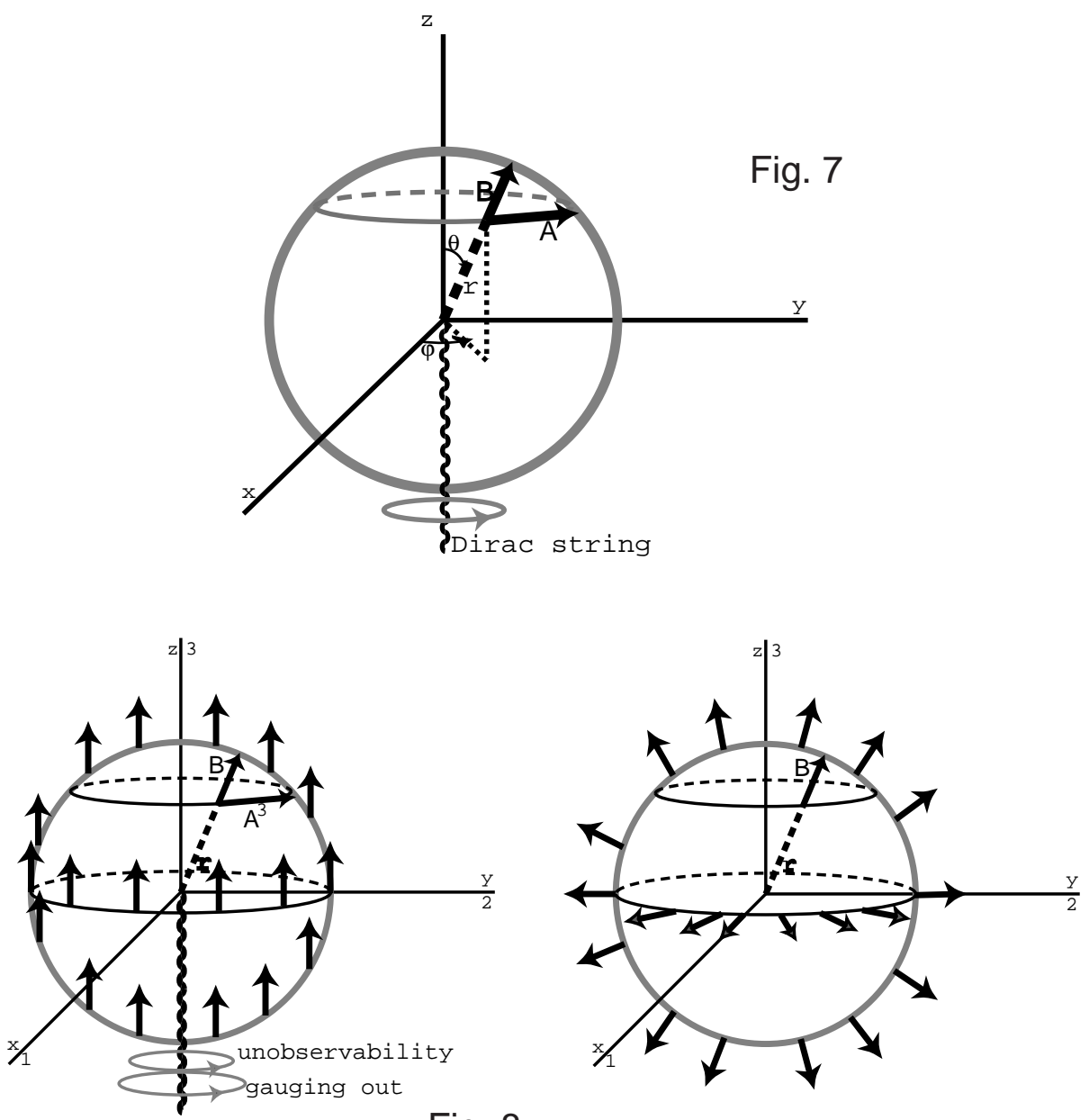

Fig. 8

Breaking the symmetry to $U(1)$ by a BEH field belonging to the adjoint group $S O(3)$ one can remove the point singularity to get the topologically stable 't Hooft-Polyakov regular monopole [114].

This procedure can be extended to Lie groups $\mathscr{G}$ of higher rank [15]. For a general Lie group $\mathscr{G}$, the possibility of gauging out the Dirac string depends on the global properties of $\mathscr{G}$. Namely, the mapping of a small circle surrounding the Dirac string onto $\mathscr{G}$ must be a curve continuously deformable to zero. Closed curves in $\mathscr{G}$ are characterized by $Z$ where $Z$ is the subgroup of the center of the universal covering $\tilde{\mathscr{G}}$ of $\mathscr{G}$ such that $\mathscr{G}=\tilde{\mathscr{G}} / Z$. Gauging out only occurs for the curve corresponding to the unit element of $Z$. This is the origin for the unconventional factor of 2 $(4 \pi=2.2 \pi)$ in Eq.(4.4) as $S O(3)=S U(2) / Z_{2}$.

The construction of regular monopoles has interesting conceptual implications.

The mixing between space and isospace indices in Eq.(4.4) means that the regular monopole is invariant under the diagonal subgroup of $S O(3)_{\text {space }} \times S O(3)_{\text {isospace. }}$ This implies that a bound state of a scalar of isospin $1 / 2$ with the monopole is a space-time fermion. In this way, fermions can be made out of bosons [16].

One can define regular monopoles in a limit in which the BEH-potential vanishes. These 


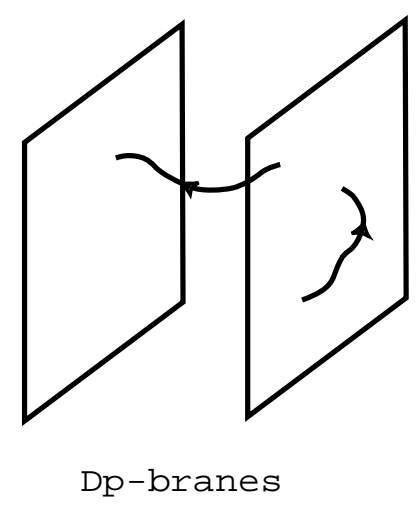

Fig. 9

are the BPS monopoles. They admit a supersymmetric extensions in which there are indications that electromagnetic duality can be realized at a fundamental level, namely that the interchange of electric and magnetic charge could be realized by equivalent but distinct actions.

The BEH-mechanism, when $\mathscr{G}$ symmetry is completely broken, is a relativistic analog of superconductivity. The latter may be viewed as a condensation of electric charges. Magnetic flux is then channeled into quantized flux tubes. In confinement, it is the electric flux which is channeled into quantized tubes. Therefore electric-magnetic duality suggests that, at some fundamental level, confinement is a condensation of magnetic monopoles and constitutes the magnetic dual of the BEH mechanism [ए7].

\subsection{A geometrical interpretation of the BEH mechanism}

The BEH mechanism operates within the context of gauge theories. Despite the fact that grand unification schemes reach scales comparable to the Planck scale, there was, a priori, no indication that Yang-Mills fields offer any insight into quantum gravity. The only approach to quantum gravity which had some success, in particular in the context of a quantum interpretation of the black holes entropies, are the superstring theory approaches and the possible merging of the five perturbative approaches (Type IIA, IIB, Type I and the two heterotic strings) into an elusive M-theory whose classical limit would be 11-dimensional supergravity. Of particular interest in that context is the discovery of Dp-branes along which the ends of open strings can move [ए8]]. This led, for the first time, to an interpretation of the area entropy of some black holes in terms of a counting of quantum states. Here we shall explain how Dp-branes yield a geometrical interpretation of the BEH mechanism.

When $N$ BPS Dp-branes coincide, they admit massless excitations from the $N^{2}$ zero length oriented strings with both end attached on the $N$ coincident branes. There are $N^{2}$ massless vectors and additional $N^{2}$ massless scalars for each dimension transverse to the branes. The open string sector has local $U(N)$ invariance. At rest, BPS Dp-branes can separate from each other in the transverse dimensions at no cost of energy. Clearly this can break the symmetry group from $U(N)$ up to $U(1)^{N}$ when all the branes are at distinct location in the transverse space, because strings joining two different branes have finite length and hence now describe finite mass excitations. The only remaining massless excitations are then due to the zero length strings with both ends on the same brane. 
This symmetry breaking mechanism can be understood as a BEH mechanism from the action describing low energy excitations of $N$ Dp-branes. This action is the reduction to $p+1$ dimensions of 10-dimensional supersymmetric Yang-Mills with $U(N)$ gauge fields [एव, [20].

The Lagrangian is

$$
\mathscr{L}=-\frac{1}{4} \operatorname{Tr} \mathbf{F}_{\mu v} \mathbf{F}^{\mu v}+\operatorname{Tr}\left(\frac{1}{2} D_{\mu} \mathbf{A}^{i} D^{\mu} \mathbf{A}^{i}-\frac{1}{4}\left[\mathbf{A}^{i}, \mathbf{A}^{j}\right]^{2}\right)+\text { fermions },
$$

where $\mu$ labels the $p+1$ brane coordinates and $i$ the directions transverse to the branes. $\mathbf{F}_{\mu \nu}=$ $F_{\mu \nu}^{a} \mathbf{T}^{a}, \mathbf{A}^{i}=A^{a i} \mathbf{T}^{a}$ where $\mathbf{T}^{a}$ is a generator of $U(N)$ in a defining representation.

The states of zero energy are given classically, and hence in general because of supersymmetry, by all commuting $\mathbf{A}^{i}=\left\{x_{m n}^{i}\right\}$ matrices, that is, up to an equivalence, by all diagonal matrices $\left\{x_{m n}^{i}\right\}=\left\{x_{m}^{i} \boldsymbol{\delta}_{m n}\right\}$. Label the $N^{2}$ matrix elements of $\mathbf{A}_{\mu}$ by $A_{\mu m n}$. The $\left(N^{2}-N\right)$ gauge fields given by the non diagonal elements $m \neq n$ acquire a mass

$$
m_{m n}^{2} \propto\left(\vec{x}_{m}-\vec{x}_{n}\right)^{2},
$$

if $\vec{x}_{m} \neq \vec{x}_{n}$, as is easily checked by computing the quadratic terms in $A_{\mu m n}$ appearing in the covariant derivatives $\operatorname{Tr} D_{\mu} \mathbf{A}^{i} D^{\mu} \mathbf{A}^{i}$.

This symmetry breaking is induced by the expectation values $\left\{x_{m}^{i}\right\}$. The gauge invariance is ensured, as usual, by unobservable $\left(N^{2}-N\right)$ NG bosons. To identify the latter we consider the scalar potential in Eq.(4.5)), namely

$$
V=\operatorname{Tr} \frac{1}{4}\left[\mathbf{A}^{i}, \mathbf{A}^{j}\right]\left[\mathbf{A}^{i}, \mathbf{A}^{j}\right]=\frac{1}{4} \sum_{i, j ; m, n}\left\langle m\left|\left[\mathbf{A}^{i}, \mathbf{A}^{j}\right]\right| n\right\rangle\left\langle n\left|\left[\mathbf{A}^{i}, \mathbf{A}^{j}\right]\right| m\right\rangle .
$$

We write

$$
\left\langle m\left|\mathbf{A}^{j}\right| n\right\rangle=x_{m}^{j} \boldsymbol{\delta}_{m n}+y_{m n}^{j} .
$$

Here the diagonal elements $\left\{x_{m}^{j}\right\}$ are the BEH expectation values and the $y_{m n}^{j}\left(=-\left[y_{n m}^{j}\right]^{*}\right)$ define $d\left(N^{2}-N\right)$ hermitian scalar fields $\left(y_{m n}^{i}\right)^{a}(a=1,2)$ where $y_{m n}^{j}=\left(y_{m n}^{j}\right)^{1}+i\left(y_{m n}^{j}\right)^{2}, m>n$, and $d$ is the number of transverse space dimensions. The mass matrix for the fields $\left(y_{m n}^{i}\right)^{a}$ is

$$
\frac{\partial^{2} V}{\partial\left(y_{m n}^{k}\right)^{a} \partial\left(y_{m n}^{l}\right)^{b}}=\delta^{a b}\left[\left(\vec{x}_{m}-\vec{x}_{n}\right)^{2} \delta^{k l}-\left(x_{m}^{k}-x_{n}^{k}\right)\left(x_{m}^{l}-x_{n}^{l}\right)\right],
$$

and has for each pair $m, n(m<n)$, two zero eigenvalues corresponding to the eigenvectors $\left(y_{m n}^{l}\right)^{a} \propto$ $\left(x_{m}^{l}-x_{n}^{l}\right)$. These are the required $\left(N^{2}-N\right)$ NG bosons, as can be checked directly from the coupling of $\mathbf{A}^{i}$ to $\mathbf{A}_{\mu}$ in the Lagrangian Eq.(4.5) .

As mentioned above, the breaking of $U(N)$ up to $U(1)^{N}$ may be viewed in the string picture as due to the stretched strings joining branes separated in the dimensions transverse to the branes. One identifies the $\left\{x_{m}^{i}\right\}$ as coordinates transverse to the brane $m$. The mass of the vector meson $A_{\mu m n}$ is then the mass shift due to the stretching of the otherwise massless open string vector excitations. The unobservable NG bosons $\vec{y}_{m n} \|\left(\vec{x}_{m}-\vec{x}_{n}\right)$ are the field theoretic expression of the unobservable longitudinal modes of the strings joining the branes $m$ and $n$. In this way Dp-branes provide a geometrical interpretation of the BEH mechanism. 
It may be worth mentioning the interesting situation which occurs when $p=0$ [지, 2]]. The Lagrangian Eq.(4.5) then describes a pure quantum mechanical system where the $\left\{x_{m n}^{i}\right\}$ are the dynamical variable. The time component $\mathbf{A}_{t}$ which enters the covariant derivative $D_{t} \mathbf{A}^{i}$ can be put equal to zero, leaving a constraint which amounts to restrict the quantum states to singlets of $S U(N)$. The $\left\{x_{m}^{i}\right\}$ which define in string theory D0-brane coordinates (viewed as partons in the infinite momentum frame in reference [2] ) are the analog, for $p=0$, of the BEH expectation values in the $p \neq 0$ case, although they label now classical collective position variables of the quantum mechanical system and not vacuum expectation values. The nondiagonal quantum degrees of freedom $\vec{y}_{m n} \perp\left(\vec{x}_{m}-\vec{x}_{n}\right)$ have a positive potential energy proportional to the distance squared between the D0-branes $m$ and $n$. Hence they get locked in their ground state when the D0-branes are largely separated from each other. In this way, the D0-brane $\mathbf{A}^{i}=\left\{x_{m n}^{i}\right\}$ matrices commute at large distance scale and define geometrical degrees of freedom. However these matrices do not commute at short distances where the potential energies of the $y_{m n}^{i}$ go to zero. This suggests that the space-time geometry exhibits non commutativity at small distances, a feature which may well turn out to be an essential element of quantum gravity.

\section{Remarks}

Physics, as we know it, is an attempt to interpret the apparent diversity of natural phenomena in terms of general laws. By essence then, it incites one towards a quest for unifying diverse physical laws.

Originally the BEH mechanism was conceived to unify the theoretical description of long range and short range forces. The success of the electroweak theory made the mechanism a candidate for further unification. Grand unification schemes, where the scale of unification is pushed close to the scale of quantum gravity effects, raised the possibility that unification might also have to include gravity. This trend towards the quest for unification received a further impulse from the developments of string theory and from its connection with eleven-dimensional supergravity. The latter was then viewed as a classical limit of a hypothetical M-theory into which all perturbative string theories would merge. In that context, the geometrization of the $\mathrm{BEH}$ mechanism is suggestive of the existence of an underlying non commutative geometry.

\section{References}

[1] F. Englert and R. Brout, Phys. Rev. Lett. 13 (31 August 1964) 321.

[2] P.W. Higgs, Physics Letters 12 (15 September 1964) 132.

[3] P.W. Higgs, Phys. Rev. Lett. 13 (19 October 1964) 508. 9

[4] Y. Nambu, Phys.Rev.Lett. 4 (1960) 380.

[5] Y. Nambu and G. Jona-Lasinio, Phys. Rev. 122 (1961) 345; Phys. Rev. 1241961246.

[6] J. Goldstone, Il Nuovo Cimento 19 (1961) 154.

\footnotetext{
${ }^{9}$ References of the original work on the BEH theory have been fully dated. The dates given here are the publication dates; submission dates follow the same sequence.
} 
[7] J. Goldstone, A. Salam and S. Weinberg, Phys. Rev. 127 (1962) 965.

[8] S. Elitzur, Phys. Rev. D12 (1975) 3978.

[9] F. Englert, R.Brout and M. Thiry, Il Nuovo Cimento 43A (1966) 244; see also the Proceedings of the 1997 Solvay Conference, Fundamental Problems in Elementary Particle Physics, Interscience Publishers J. Wiley ans Sons, p 18.

[10] For a detailed history on this subject, see M. Veltman "The path to renormalizability”, invited talk at the Third International Symposium on the History of Particle Physics, June 24-27, 1992; (Printed in Hoddeson and al. , 1997).

[11] S.L. Glashow, Nucl. Phys. B22 (1961) 579; S. Weinberg, Phys. Rev. Lett. 19 (1967) 1264; A. Salam, in Elementary Particle Physics ed. N. Svartholm (Amquist and Wiksels, Stockholm, 1969).

[12] H. Georgi, H.R. Quinn and S. Weinberg, Phys. Rev. Lett. 333 (1974) 1974.

[13] P.A.M. Dirac, Phys. Rev. 74 (1948) 817.

[14] G 't Hooft, Nucl. Phys. B79 (1974) 276; A.M. Polyakov, ZhETF 20 (1974) 403, (JETP Lett. 20 (1974) 199).

[15] F. Englert and P. Windey, Phys. Rev. D14 (1976) 2728; P. Goddard, J. Nuyts and D. Olive, Nucl. Phys. B125 (1977) 1.

[16] R. Jackiw and C. Rebbi, Phys. Rev. Lett. 36 (1976) 1116; P. Hasenfratz and G. 't Hooft, Phys. Rev. Lett. 36 (1976) 1119; A. Goldhaber, Phys. Rev. Lett. 36 (1976) 1122.

[17] N. Seiberg and E.Witten, Nucl. Phys. B431 (1994) 484, hep-th/9408099, and references therein.

[18] J. Dai, R.G. Leigh and J. Polchinski, Mod. Phys. Lett. A4 (1989) 2073.

[19] J. Polchinski, Phys. Rev. D50 (1994) 6041.

[20] E. Witten, Nucl. Phys. B460 (1996) 335, hep-th/9510135.

[21] T. Banks, W. Fischler, S.H. Shenker and L. Susskind, hep-th/9610043. 